\title{
Iguratimod prevents ovariectomy-induced bone loss and suppresses osteoclastogenesis via inhibition of peroxisome proliferator-activated receptor- $\gamma$
}

\author{
YING-XING WU ${ }^{1}$, YUE SUN ${ }^{2}$, YA-PING YE ${ }^{1}$, PENG ZHANG $^{2}$, JIA-CHAO GUO ${ }^{1}$, \\ JUN-MING HUANG ${ }^{1}$, XING-ZHI JING ${ }^{1}$, WEI XIANG ${ }^{1}$, SHI-YING YU ${ }^{2}$ and FENG-JING GUO ${ }^{1}$ \\ ${ }^{1}$ Department of Orthopedics and ${ }^{2}$ Cancer Center, Tongji Hospital, Tongji Medical College, \\ Huazhong University of Science and Technology, Wuhan, Hubei 430030, P.R. China
}

Received March 17, 2017; Accepted September 21, 2017

DOI: $10.3892 / \mathrm{mmr} .2017 .7648$

\begin{abstract}
Iguratimod is known for its anti-inflammatory activities and therapeutic effects in patients with rheumatoid arthritis. It has previously been demonstrated that iguratimod attenuates bone destruction and osteoclast formation in the Walker 256 rat mammary gland carcinoma cell-induced bone cancer pain model. Therefore, it was hypothesized that iguratimod may additionally exhibit therapeutic effects on benign osteoclast-associated diseases including postmenopausal osteoporosis. In the present study, ovariectomized mice were used to investigate the effects of iguratimod in vivo. Bone marrow mononuclear cells were cultured to detect the effects of iguratimod on receptor activator of nuclear factor- $\mathrm{\kappa B}$ ligand (RANKL)-induced osteoclastogenesis in vitro and the molecular mechanisms involved. It was demonstrated that iguratimod may prevent ovariectomy-induced bone loss by suppressing osteoclast activity in vivo. Consistently, iguratimod may inhibit RANKL-induced osteoclastogenesis and bone resorption in primary bone marrow mononuclear cells. At the molecular level, peroxisome proliferator-activated receptor- $\gamma($ PPAR- $\gamma) / \mathrm{c}-$ Fos pathway, which is essential in RANKL-induced osteoclast differentiation, was suppressed by iguratimod. Subsequently, iguratimod decreased the expression of nuclear factor of activated $\mathrm{T}$ cells $\mathrm{cl}$ and downstream osteoclast marker genes. The results of the present study demonstrated that iguratimod may inhibit ovariectomy-induced bone loss and osteoclastogenesis by modulating RANKL signaling. Therefore, iguratimod may act as a novel therapeutic to prevent postmenopausal osteoporosis.
\end{abstract}

Correspondence to: Dr Feng-Jing Guo, Department of Orthopedics, Tongji Hospital, Tongji Medical College, Huazhong University of Science and Technology, 1095 Jiefang Avenue, Wuhan, Hubei 430030, P.R. China

E-mail: guofjdoc@163.com

Key words: iguratimod, postmenopausal osteoporosis, ovariectomy, RANKL, PPAR- $\gamma$

\section{Introduction}

Bone homeostasis is maintained by repeated cycles of bone formation and bone resorption in a well-balanced process called bone remodeling (1). However, many bone metabolism diseases can disturb the balance in the bone remodeling process. Postmenopausal osteoporosis is one such systemic skeletal disease characterized by low bone density and micro architectural deterioration that lead to increased bone fragility thus making the bone susceptible to fracture $(2,3)$. Moreover, lack of functional ovaries leads to a decline in estrogen levels that increases bone formation and, to a much higher extent, increases bone resorption, leading to net bone loss (4). Estrogen deficiency may also result in the over expression of receptor activator of nuclear factor- $\mathrm{KB}(\mathrm{NF}-\mathrm{\kappa} \mathrm{B})$ ligand (RANKL) by B lymphocytes (5), which may in part explain the excessive osteoclasts formation and trabecular bone loss in postmenopausal osteoporosis patients.

Osteoclasts are multinucleated giant cells differentiated from monocyte-macrophage lineage precursor cells, and possess a unique ability to resorb bones. Osteoclast differentiation is primarily governed by 2 key cytokines, macrophage colony-stimulating factor (M-CSF) and RANKL. M-CSF supports cell survival and proliferation while RANKL serves as a signal for osteoclastogenesis $(6,7)$. The binding of RANKL and its receptor RANK on osteoclast precursor cells activates downstream pathways including peroxisome proliferator-activated receptor- $\gamma$ (PPAR- $\gamma$ ) and c-Fos (8), which increase the expression of nuclear factor of activated $\mathrm{T}$ cells $\mathrm{c} 1$ (NFATc1) (9). NFATc1 is a master factor that activates the expression of osteoclast marker genes and subsequently results in enhanced differentiation and function of osteoclasts (10-12). Taking together, interfering with these pathways may help prevent pathologically enhanced osteoclasts formation and bone loss.

Iguratimod (T-614), an efficacious and safe anti-rheumatoid arthritis drug, is reported to exert its therapeutic effect by reducing the production of inflammatory cytokines such as interleukin (IL)-1 $\beta$, IL-6, IL-8 and tumor necrosis factor (TNF)- $\alpha(13,14)$. In type II collagen-induced arthritis and spontaneous arthritis models, iguratimod can reduce joint 
destruction and bone resorption (15). However, whether iguratimod could suppress osteoclasts formation and bone loss in postmenopausal osteoporosis animal models has not been verified. Furthermore, in vivo and in vitro studies showed that PPAR- $\gamma$ is essential in RANKL-induced osteoclast differentiation through direct regulation of $\mathrm{c}-$ Fos expression $(8,16)$. Whether PPAR- $\gamma$ is a target of iguratimod in osteoclastogenesis should be further explored. Therefore, in the present study, we investigated the effects of iguratimod on bone loss and osteoclasts formation in ovariectomized mice models and in primary bone marrow mononuclear cells (BMMCs) models, and elucidated the underlying molecular mechanisms.

\section{Materials and methods}

Reagents and antibodies. Iguratimod was provided by Simcere Pharmaceutical Research Co., Ltd. (Jiangsu, China). The drug was suspended in $0.5 \%$ methylcellulose solution for in vivo use, and dissolved in DMSO (Sigma-Aldrich; Merck KGaA, Darmstadt, Germany) for in vitro use. Recombinant soluble mouse M-CSF and RANKL were obtained from PeproTech (Rocky Hill, NJ, USA). Rabbit antibody against NFATc1 (no. 8032, dilution 1:1,000) was purchased from Cell Signaling Technology, Inc. (Danvers, MA, USA). Rabbit antibody against c-Fos (no. sc-52, dilution 1:200) was purchased from Santa Cruz Biotechnology, Inc. (Santa Cruz Biotechnology, Inc., Dallas, TX, USA). Rabbit antibody against PPAR- $\gamma$ (no. BA1693-2, dilution 1:200) and mouse antibody against GAPDH (no. BM1623, dilution 1:200) were purchased from Wuhan Boster Biological Technology, Ltd. (Wuhan, China). Rosiglitazone was purchased from Abcam (Cambridge, MA, USA).

Animals. Twelve-weeks-old C57/BL6 female mice (29 \pm 2 g) (Experimental Animal Center of Tongji Hospital, Wuhan, China) were maintained at a constant temperature of $25^{\circ} \mathrm{C}$ under a 12-h light/12-h dark cycle with free access to food and water. All experimental protocols were approved by the Medical Ethics Committee of Huazhong University of Science and Technology and were performed according to the ethical guidelines of the National Institutes of Health Guide for Care and Use of Laboratory Animals.

Animals were divided randomly into 3 groups $(n=10$ mice/group): Sham-operated mice treated with $0.5 \%$ methylcellulose solution (vehicle) (SHAM), bilateral ovariectomized mice treated with vehicle $(\mathrm{OVX})$ and bilateral ovariectomized mice treated with iguratimod $(30 \mathrm{mg} / \mathrm{kg} / \mathrm{day})$ (OVX+T-614) (17). Ovariectomy was performed as previously described (18). Briefly, mice were anesthetized by intraperitoneally injecting pentobarbital sodium at a dose of $50 \mathrm{mg} / \mathrm{kg}$ body weight. Bilateral ovaries were removed through a dorsal approach. All treatments began on day 1 after operation and were administered orally. After 6 weeks, mice were sacrificed by an overdose of anesthesia to isolate the femurs and uterus for use in the following experiments.

Bone structure analysis. The distal femoral bone structure was analysed with a micro-computed tomography ( $\mu$-CT) system ( $\mu$-CT50; Scanco Medical, Bassersdorf, Switzerland). Scans were obtained at $100 \mathrm{kV}$ and $98 \mu \mathrm{A}$; the resolution was set to
$10.5 \mu \mathrm{m}$. 3D reconstruction were analyzed using the built-in software in the $\mu$-CT system. Trabecular structural parameters including bone volume/tissue volume (BV/TV), structure model index (SMI), trabecular number (Tb.N) and trabecular separation ( $\mathrm{Tb}$. $\mathrm{Sp}$ ) were also evaluated.

Histological analysis. For histological analysis, femur samples were fixed in $4 \%$ paraformaldehyde for $24 \mathrm{~h}$, decalcified in $10 \%$ ethylenediaminetetraacetic acid (EDTA) solution for 3 weeks and embedded in paraffin wax. Hematoxylin and eosin (H\&E) staining was performed to observe the trabecular structure. Tartrate-resistant acid phosphatase (TRAP) staining (Sigma-Aldrich; Merck KGaA) was performed following standard protocols and the numbers of osteoclasts near femoral metaphysis were counted (19). Images were obtained using Leica Microsystems (Wetzlar, Germany).

Serum biochemistry. For serum biochemical analysis, a retro-orbital puncture was performed immediately prior to euthanasia to collect blood. Blood was collected from each mouse and plasma was separated by centrifugating for $15 \mathrm{~min}$ at $1,000 \mathrm{x} \mathrm{g}$ in room temperature. Serum levels of type 1 collagen cross-linked C-terminal telopeptide (CTX-I) were measured with ELISA kits (Nordic Bioscience Diagnostics A/S, Herlev, Denmark) according to the manufacturer's instructions.

Cell cultures. BMMCs were obtained from 6-weeks-old C57BL/6 mice as previously described $(20,21)$. Briefly, marrow cavities of isolated femurs and tibias were exposed and flushed with $\alpha$-minimum essential medium ( $\alpha$-MEM; Gibco; Thermo Fisher Scientific, Inc., Waltham, MA, USA). Cells were then collected and cultured in $\alpha$-MEM with $10 \%$ fetal bovine serum (FBS; Gibco; Thermo Fisher Scientific, Inc.), $100 \mathrm{U} / \mathrm{ml}$ penicillin, $100 \mu \mathrm{g} / \mathrm{ml}$ streptomycin (Gibco; Thermo Fisher Scientific, Inc.) and M-CSF (30 ng/ml). After $24 \mathrm{~h}$, non-adherent cells were collected and supplemented with M-CSF (30 ng/ml). After 3 days, adherent cells were seeded in different plates for use in the following experiments.

Cell Counting Kit-8 (CCK-8) assay. Cell viability was assessed using a CCK-8 assay (Boster Biological Technology, Ltd.) according to the manufacturer's instructions. Briefly, BMMCs were seeded at a density of 5,000 cells/well in 96-well plates. After $24 \mathrm{~h}, \mathrm{BMMCs}$ were treated with phosphate-buffered saline (PBS), 0 (vehicle), $0.3,3$ or $30 \mu \mathrm{g} / \mathrm{ml}$ iguratimod in the presence of M-CSF (30 ng/ml). After 1, 3 and 5 days, medium containing $10 \% \mathrm{CCK}-8$ was added to each well and then incubated in darkness at $37^{\circ} \mathrm{C}$ for $1 \mathrm{~h}$. The absorbance was measured on an ELX800 absorbance microplate reader (Bio-Tek Instruments Inc., Winooski, VT, USA) at a wavelength of $450 \mathrm{~nm}$.

In vitro osteoclastogenesis assay. BMMCs were plated in 96-well plates at a density of 10,000 cells/well and cultured with M-CSF (30 ng/ml) and RANKL (50 ng/ml) in the presence of vehicle or various concentrations of iguratimod. After 5 days, TRAP staining was performed according to the manufacturer's instructions. Images were obtained and 
TRAP-positive multinucleated ( $>3$ nuclei) cells were counted as osteoclasts.

Bone pit formation by osteoclasts. BMMCs were seeded at a density of 20,000 cells/well in a Corning Osteo Assay Surface plate (Corning Inc., Corning, NY, USA). Cells were cultured with M-CSF (30 ng/ml) and RANKL (100 ng/ml) for 7 days, then treated with vehicle or various concentrations of iguratimod for an additional 5 days. Then, the plate was washed with $5 \%$ sodium hypochlorite for $5 \mathrm{~min}$. Images of bone resorption were captured and quantified.

RNA extraction, reverse transcription and real-time quantitative PCR. BMMCs were seeded at a density of $1 \times 10^{5}$ cells $/ \mathrm{mm}^{2}$ in 6 -well plates. Cells were cultured with $\mathrm{M}-\mathrm{CSF}(30 \mathrm{ng} / \mathrm{ml})$ and RANKL $(50 \mathrm{ng} / \mathrm{ml})$ in the presence of vehicle or $3 \mu \mathrm{g} / \mathrm{ml}$ iguratimod for 5 days. Then total RNA was extracted from BMMCs using TRIzol reagent (Invitrogen Life Technologies, Carlsbad, CA, USA) as previously described (21). First-strand cDNA was synthesized using ReverTra Ace qPCR RT kit (Toyobo Co., Ltd., Osaka, Japan) to perform RT-qPCR using the Thunderbird SYBR qPCR Mix (Toyobo Co., Ltd.) and a Bio-Rad Q5 instrument (Bio-Rad Laboratories, Inc., Hercules, CA, USA). All reactions were performed according to the manufacturer's instructions, and target gene expression was normalized to the reference gene glyceraldehyde 3-phosphate dehydrogenase (GAPDH). The relative expression levels of each gene were calculated using the comparative $2^{-\Delta \Delta C t}$ method (22). The primers used for RT-qPCR are listed in Table I.

Western blot analysis. BMMCs were seeded at a density of $1 \times 10^{5}$ cells/well in 6-well plates. To detect effect of iguratimod on c-Fos and NFATc1 expression, cells were cultured with M-CSF (30 ng/ml) and RANKL (50 $\mathrm{ng} / \mathrm{ml})$ in the presence of vehicle or $3 \mu \mathrm{g} / \mathrm{ml}$ iguratimod for 2 days and 5 days. To detect the crosstalk between iguratimod and PPAR- $\gamma$, cells were cultured with M-CSF and RANKL in the presence of vehicle, $3 \mu \mathrm{g} / \mathrm{ml}$ iguratimod or $1 \mu \mathrm{M}$ rosiglitazone for 5 days.

Cell lysates were prepared with the RIPA Lysis Buffer (Boster Biological Technology, Ltd.) containing $1 \mathrm{mM}$ phenylmethanesulfonyl fluoride (PMSF; Boster Biological Technology, Ltd.). The lysates were centrifuged for $20 \mathrm{~min}$ at $12,000 \mathrm{x}$. Then supernatants were collected. Protein concentration of each sample was detected using BCA protein assay (no. AR0146; Boster Biological Technology, Ltd.) according to the standard protocol. Western blot analysis was then performed as described $(21,23)$. In brief, total cell proteins were separated on $10 \%$ SDS-PAGE and transferred to polyvinylidene fluoride (PVDF) membranes (Millipore, Billerica, MA, USA). Subsequently, membranes were blocked with $5 \%$ bovine serum albumin (BSA) and immunoblotted with corresponding primary antibody overnight at $4^{\circ} \mathrm{C}$. Then, the membranes were incubated with appropriate horseradish peroxidase-labelled secondary antibody (nos. BA1001 or BA1003, dilution 1:2,000; Boster Biological Technology, Ltd.) for $1 \mathrm{~h}$ at room temperature. Immunoreactivity was detected with enhanced chemiluminescence (Boster Biological Technology, Ltd.) and images were taken by ChemiDoc ${ }^{\mathrm{TM}} \mathrm{XRS}+$ System with Image Lab ${ }^{\mathrm{TM}}$ software (Bio-Rad Laboratories, Inc.).
Table I. Primers used in RT-qPCR.

Genes $\quad$ Primers (5'-3')

\begin{tabular}{ll}
\hline NFATc1 & \\
F & CAACGCCCTGACCACCGATAG \\
R & GGGAAGTCAGAAGTGGGTGGA
\end{tabular}

TRAP

$\mathrm{F}$

$\mathrm{R}$

TACCTGTGTGGACATGACC

CAGATCCATAGTGAAACCGC

Cathepsin K

$\mathrm{F}$

$\mathrm{R}$

TGTATAACGCCACGGCAAA

MMP-9

$\mathrm{F}$

$\mathrm{R}$

c-Fos

$\mathrm{F}$

$\mathrm{R}$

GGTTCACATTATCACGGTCACA

TCCAGTACCAAGACAAAGCCTA
TTGCACTGCACGGTTGAA

GGTGAAGACCGTGTCAGGAG

GAPDH

F

CTCCCACTCTTCCACCTTCG

R TTGCTGTAGCCGTATTCATT

F, forward; R, reverse; GAPDH, glyceraldehyde 3-phosphate dehydrogenase; TRAP, tartrate resistant acid phosphatase; NFATc1, nuclear factor of activated T cells 1; MMP-9, matrix metalloproteinase-9.

Statistical analysis. All quantitative data are expressed as means \pm SD. Statistical analysis between 2 groups was performed using Student's t-test. Statistical comparison of more than 2 groups was performed using one-way analysis of variance (ANOVA) followed by a Tukey's test. $\mathrm{P}<0.05$ was considered to indicate a statistically significant difference.

\section{Results}

Effect of iguratimod on OVX-induced bone loss. Six weeks after operation, mice in three groups were sacrificed. We used $\mu$-CT scanning to analyze the trabecular bone changes in distal femoral metaphyses of mice. The results demonstrated significant decrease in trabecular BV/TV and Tb. $\mathrm{N}$ and increase in SMI and Tb. Sp in the OVX group when compared with the SHAM group. Compared with the OVX group, treatment of OVX mice with iguratimod significantly attenuated trabecular bone loss revealed by changes in histomorphometric parameters (Fig. 1A and B). Furthermore, mice in the OVX and OVX+T-614 groups exhibited a marked decrease in the wet weight of uterus (Fig. 1C) compared with the SHAM group, suggesting the success of ovariectomy.

H\&E staining of the femoral sections were then used to further corroborate these results. Trabeculae in the OVX group were rare and thin in regions proximal and distal to the growth plate. Treatment with iguratimod significantly 

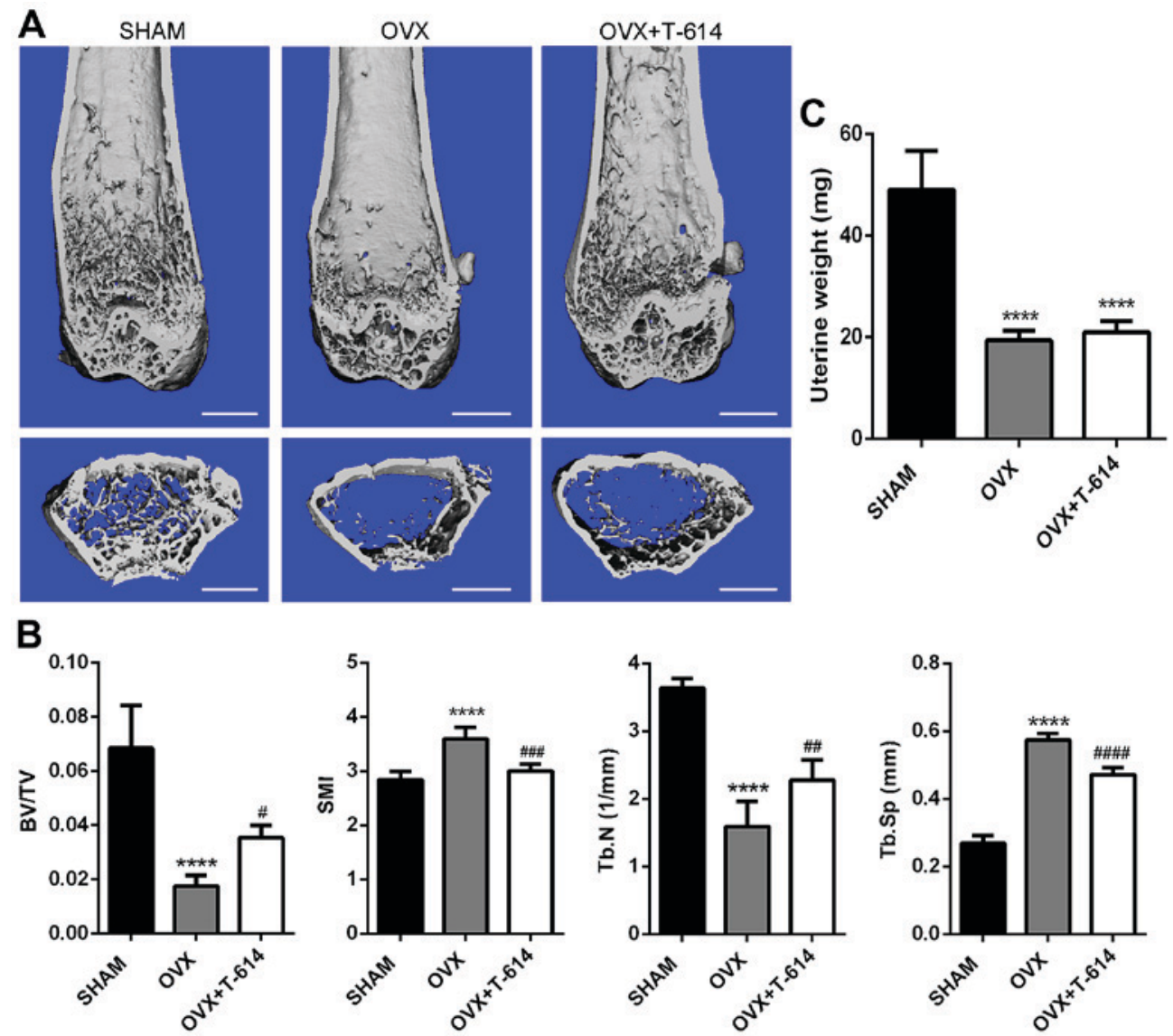

Figure 1. Iguratimod alleviates bone loss in ovariectomized mice. (A) $\mu$-CT images of distal femurs from representative specimens from the SHAM, OVX and OVX+T-614 groups. Scale bar, $1 \mathrm{~mm}$. (B) Histograms represent the 3D trabecular structural parameters of the distal femur: Trabecular BV/TV, SMI, Tb.N and Tb.Sp. (C) Mice uterus was isolated and weighed. Data are presented as means \pm SD. $n=10{ }^{* * * * *} \mathrm{P}<0.0001$ vs. SHAM; ${ }^{\#} \mathrm{P}<0.05,{ }^{\# \#} \mathrm{P}<0.01,{ }^{\# \# \#} \mathrm{P}<0.001,{ }^{\# \# \# "} \mathrm{P}<0.0001$ vs. OVX. SHAM, sham operated and vehicle treated mice; OVX, bilateral-ovariectomized and vehicle treated mice; T-614, bilateral-ovariectomized and iguratimod treated mice; BV/TV, bone volume/tissue volume; SMI, structure model index; Tb. N, trabecular number; Tb. Sp, trabecular separation.

increased trabecular density and thickness when compared to the OVX group (Fig. 2A).

We then stained femoral sections with TRAP to investigate the effects of iguratimod on osteoclasts differentiation. Mice in the OVX+T-614 group had reduced numbers of TRAP-positive multinucleated cells, analyzed by the number of osteoclasts per $200 \mathrm{x}$ version, compared with mice in the OVX group (Fig. 2B and C).

Moreover, compared with the OVX group, mice in the OVX+T-614 group also displayed decreased serum levels of CTX-I, which is a biomarker of bone resorption (Fig. 2D). These results together suggest that treatment with iguratimod can attenuate OVX-induced bone loss by inhibiting the differentiation of osteoclasts.

Effect of iguratimod on RANKL-mediated osteoclastogenesis and osteoclasts function in vitro. To further explore the impact of iguratimod on osteoclastogenesis, we tested its effect on BMMCs. We first detected the potential cytotoxicity of iguratimod using the CCK-8. As shown in Fig. 3, even at $30 \mu \mathrm{g} / \mathrm{ml}$ concentration, iguratimod did not influence the viability and proliferation of BMMCs.

We then treated BMMCs with different concentrations of iguratimod $(0,0.3,3$ or $30 \mu \mathrm{g} / \mathrm{ml})$ in the presence of RANKL $(50 \mathrm{ng} / \mathrm{ml})$ and M-CSF $(30 \mathrm{ng} / \mathrm{ml})$ for 5 days. As shown in
Fig. 4, iguratimod strongly inhibited RANKL-mediated osteoclastogenesis in a dose-dependent manner. At the concentration of $3 \mu \mathrm{g} / \mathrm{ml}$ iguratimod, there were no visible TRAP-positive multinucleated cells.

To evaluate the effect of iguratimod on the bone resorption function of osteoclasts, an osteo assay surface plate was used. After seeding onto a bone slice, BMMCs were cultured with RANKL (100 ng/ml) and M-CSF (30 ng/ml) for 7 days, and then additional 5 days in the presence of different concentrations of iguratimod. As shown in Fig. 4B and D, iguratimod significantly suppressed the bone resorption function of osteoclasts.

Effect of iguratimod on RANKL-induced c-Fos, NFATcl and osteoclast marker gene expression. Stimulation of RANKL may activate a variety of transcription factors including c-Fos and downstream NFATc1. Subsequently, activated NFATc1 can increase the expression of osteoclast marker genes such as TRAP, cathepsin K and matrix metalloprotein-9 (MMP-9). We then examined the effect of iguratimod on these genes. As shown in Fig. 5A, RT-qPCR showed that incubation with RANKL significantly increased the mRNA expression of c-Fos, NFATc1, TRAP, cathepsin K and MMP-9 in BMMCs on day 5. Iguratimod drastically suppressed RANKL-induced upregulation of all these genes at the concentration of $3 \mu \mathrm{g} / \mathrm{ml}$ 

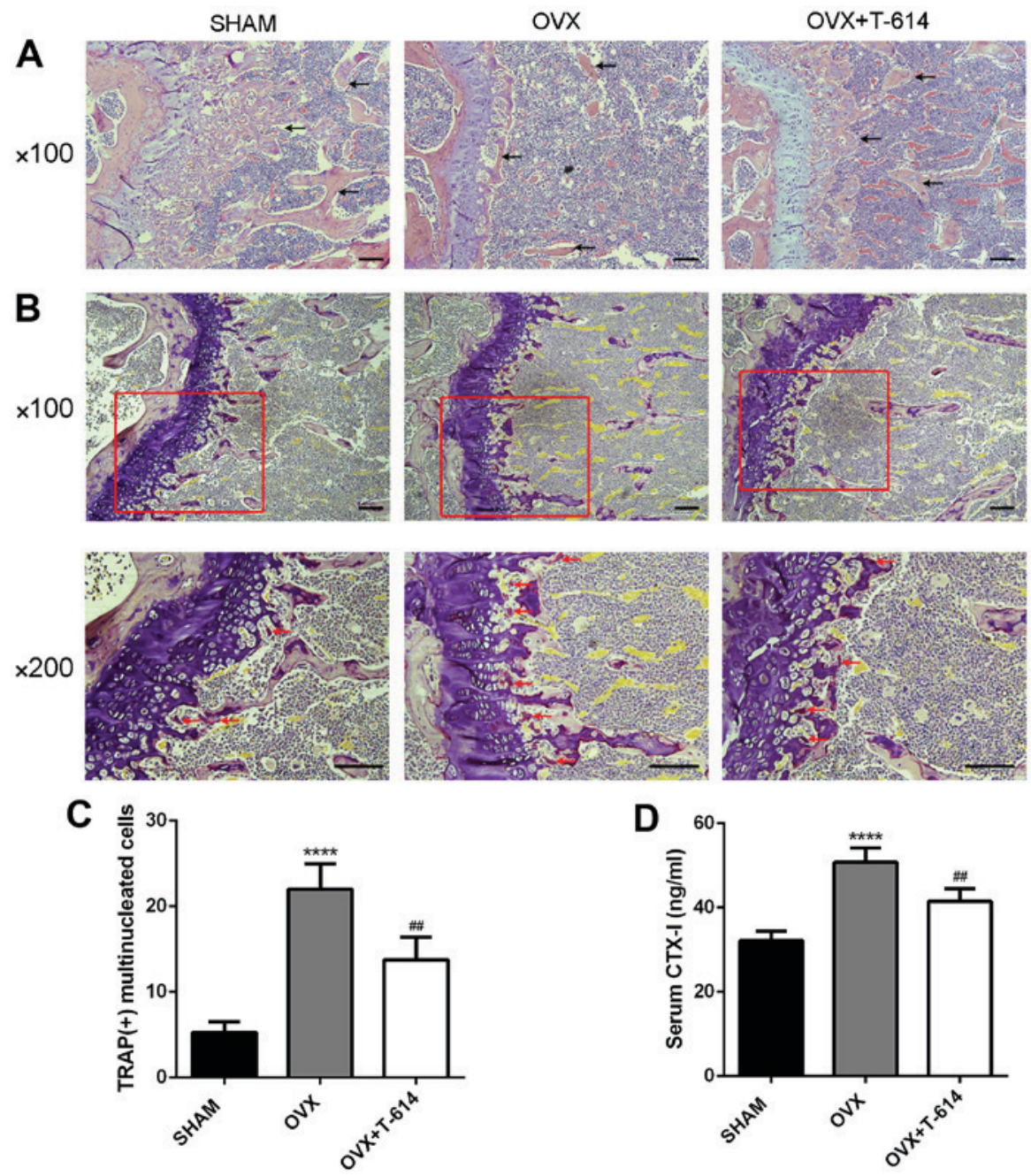

Figure 2. Iguratimod suppresses OVX-induced bone resorption and osteoclasts formation. (A) Sections of distal femurs from SHAM, OVX and OVX+T-614 groups were stained with H\&E. Bone trabeculas are indicated with black arrows. (B) TRAP-stained histological sections of the distal femurs from different groups. TRAP-positive cells are indicated with red arrows. (C) Quantitative analysis of TRAP-positive osteoclast numbers per x200 version. (D) Serum levels of CTX-I were measured using ELISA. Scale bar, $100 \mu \mathrm{m}$. Data are presented as means $\pm \mathrm{SD}$. $\mathrm{n}=10 .{ }^{* * * * *} \mathrm{P}<0.0001 \mathrm{vs}$. SHAM; ${ }^{* \# P<0.01 ~ v s . ~ O V X}$. TRAP, tartrate-resistant acid phosphatase; CTX-I, type 1 collagen cross-linked C-terminal telopeptide.

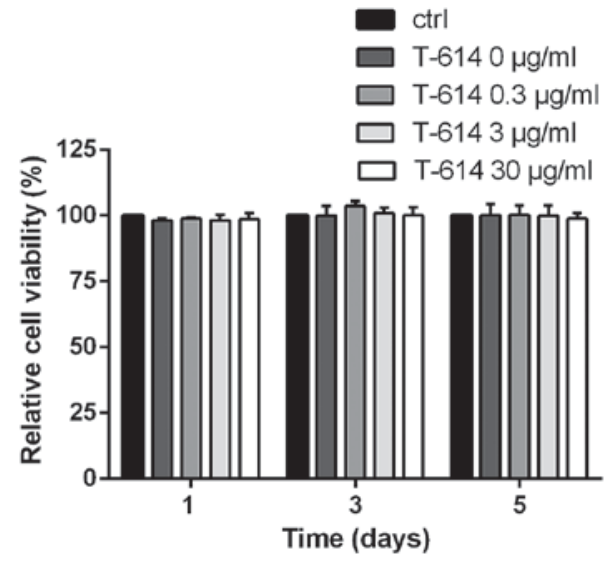

Figure 3. Iguratimod shows no influence on the viability of BMMCs. Cell viability was assessed using a CCK-8 assay and calculated using the formula: Cell viability $=\mathrm{X} / \mathrm{ctrl} \times \mathrm{x} 100 \%$. Data from 3 independent experiments are presented as means $\pm \mathrm{SD}$. SD, standard deviation.

which is close to the plasma concentration of iguratimod in rheumatoid arthritis patients treated with 50-100 mg/day (13).
The effects of iguratimod on the expression of c-Fos and NFATc1 were corroborated by immunoblotting (Fig. 5B and C).

Effect of iguratimod on PPAR- $\gamma$ signaling. PPAR- $\gamma$ plays an essential role in osteoclastogenesis though directly regulating c-Fos. Therefore, we then explored whether iguratimod suppresses osteoclastogenesis though targeting PPAR- $\gamma$ signaling. BMMCs were treated with $3 \mu \mathrm{g} / \mathrm{ml}$ iguratimod and/or $1 \mu \mathrm{M}$ rosiglitazone (BRL, an agonist of PPAR- $\gamma$ ) in the presence of RANKL and M-CSF for 5 days. As shown in Fig. 6, RANKL-induced osteoclasts formation is further promoted by rosiglitazone. Treatment of rosiglitazone could partly reverse the inhibitory effect of iguratimod.

To further validate the effect of iguratimod on the PPAR- $\gamma$ signaling, proteins were extracted and subjected to immunoblotting. Consistently, rosiglitazone partly reversed the inhibitory effect of iguratimod on the expression of PPAR- $\gamma$ (Fig. 7). The expression of downstream c-Fos and NFATc1 were also suppressed by iguratimod and partly retrieved by rosiglitazone. 

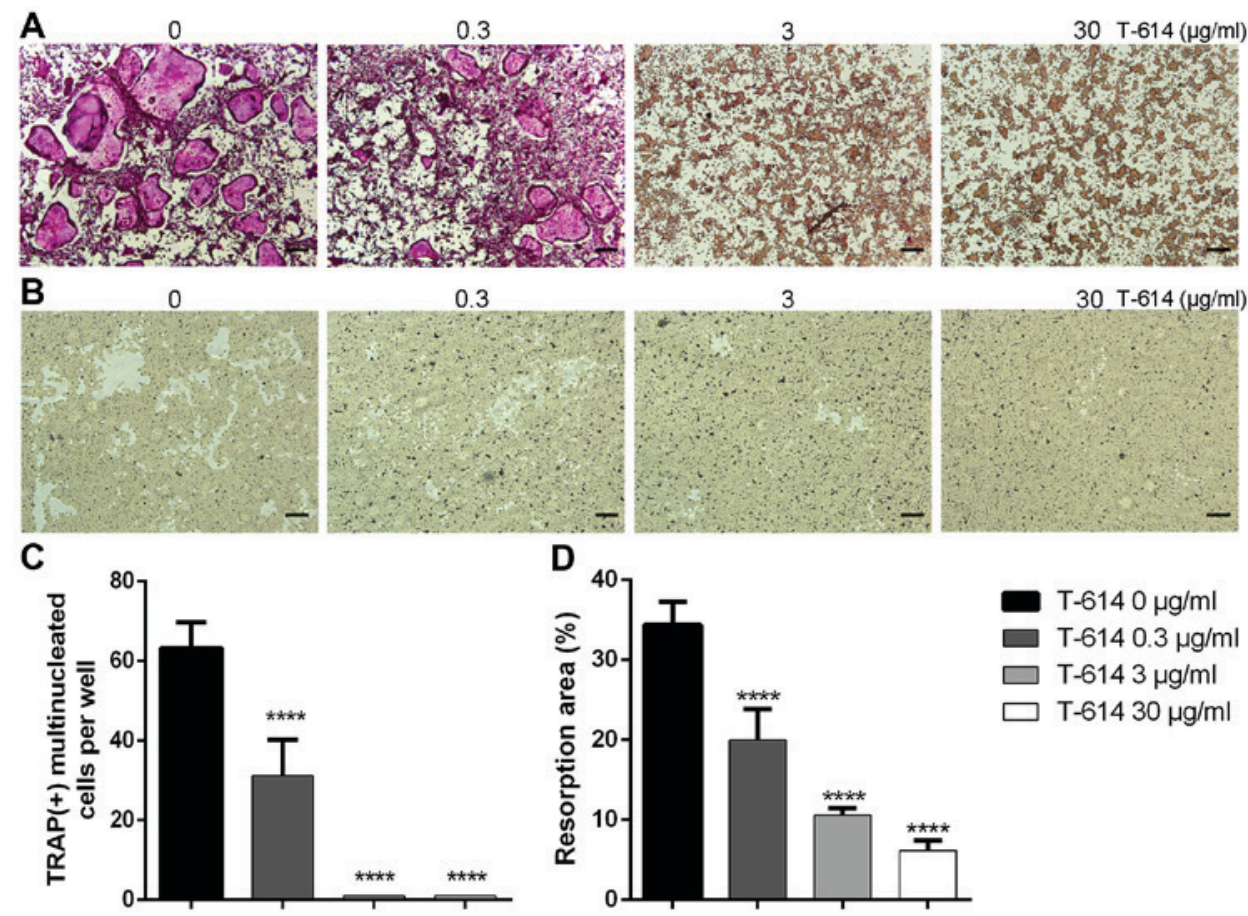

3

30 T-614 $(\mu \mathrm{g} / \mathrm{ml})$
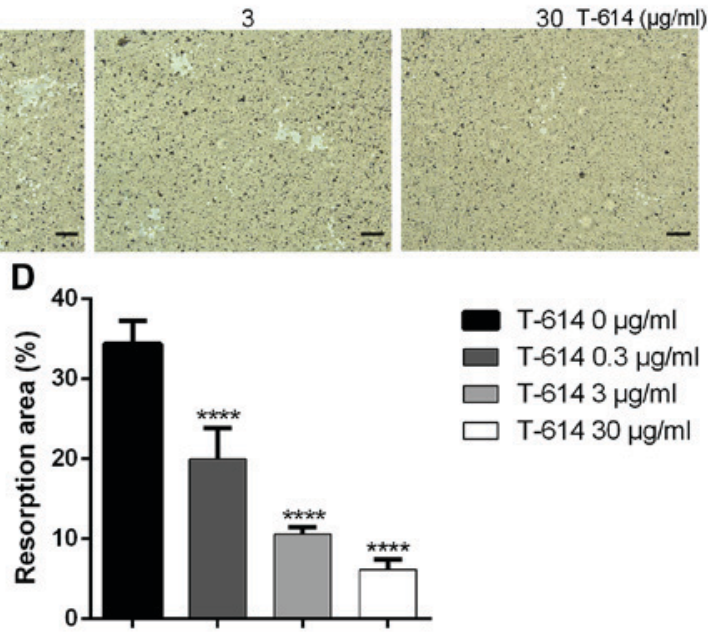

- $\mathrm{T}-6140 \mu \mathrm{g} / \mathrm{ml}$

- T-614 $0.3 \mu \mathrm{g} / \mathrm{ml}$

$\square \mathrm{T}-6143 \mu \mathrm{g} / \mathrm{ml}$

$\square \mathrm{T}-61430 \mu \mathrm{g} / \mathrm{ml}$ in each well were counted (C). (B and D) A Corning Osteo Assay Surface was used to detect the effect of iguratimod on the bone resorption function of osteoclasts. Images of bone resorption were obtained (B) and quantified using ImageJ (D). Scale bar, $200 \mu \mathrm{m}$. All data are from 3 independent experiments and are presented as means $\pm \mathrm{SD}$. ${ }^{* * * *} \mathrm{P}<0.0001$ vs. vehicle-treated controls $(\mathrm{T}-614,0 \mu \mathrm{g} / \mathrm{ml})$.
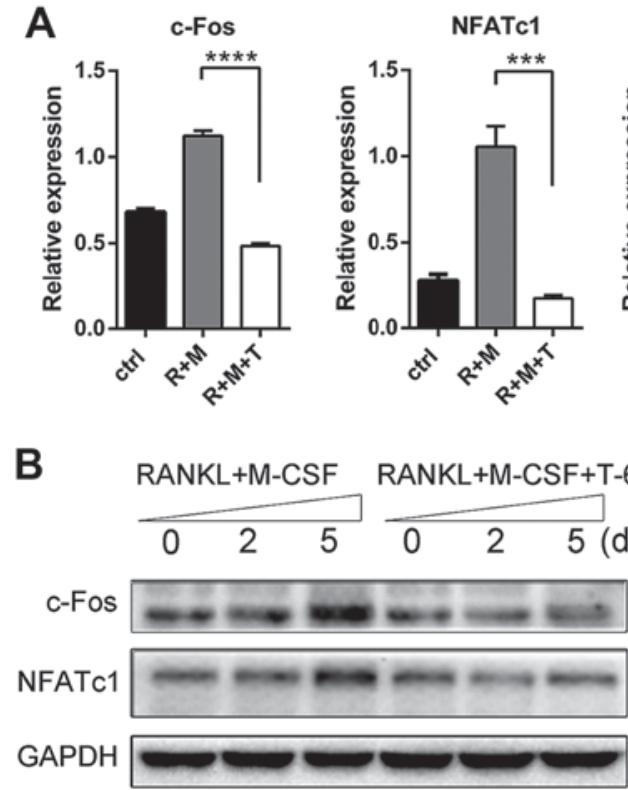
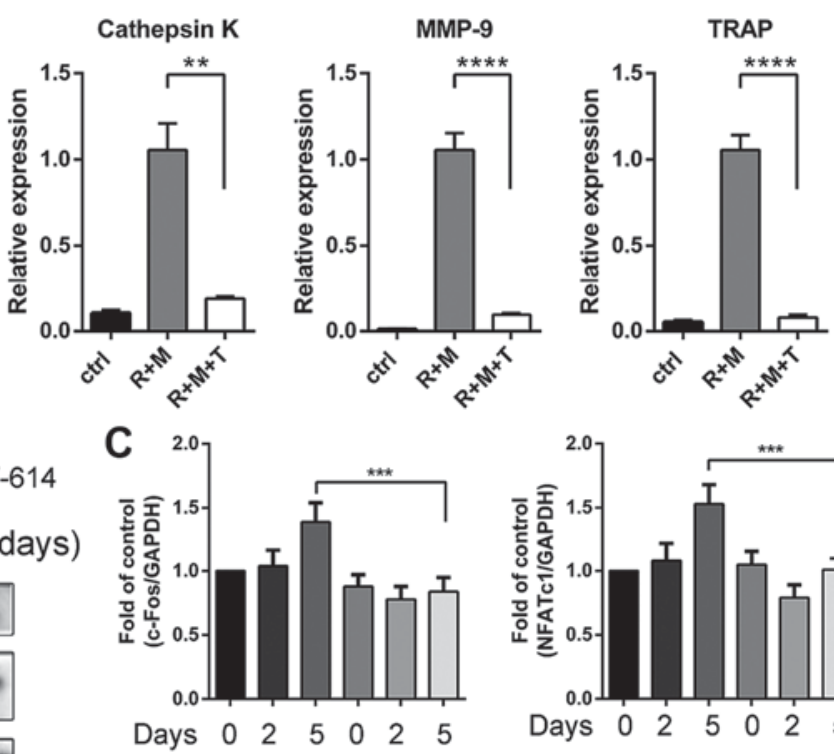

RANKL ++++++

T-614 - - +++

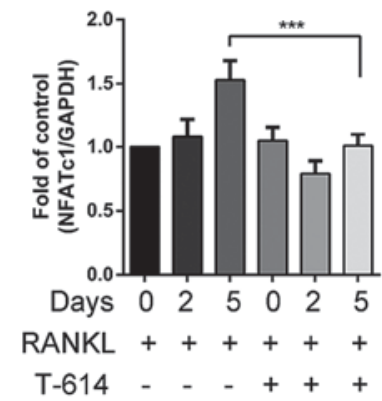

Figure 5. Iguratimod inhibits the expression of c-Fos, NFATc1 and osteoclast marker genes. (A) The mRNA levels of c-Fos, NFATc1 and osteoclast marker genes were detected using RT-qPCR. Data are presented as means \pm SD. (B and C) Proteins were extracted at indicated times and protein expression levels of c-Fos and NFATc1 were detected by western blotting (B) and quantified (C). The experiments were repeated 3 times independently. Data are presented as means $\pm \mathrm{SD} .{ }^{* *} \mathrm{P}<0.01,{ }^{* * * *} \mathrm{P}<0.001,{ }^{* * * * *} \mathrm{P}<0.0001$. NFATc1, nuclear factor of activated T cells c1; MMP-9, matrix metalloproteinase-9; TRAP, tartrate-resistant acid phosphatase; RANKL, receptor activator of nuclear factor-кB ligand; M-CSF, macrophage colony-stimulating factor; GAPDH, glyceraldehyde 3-phosphate dehydrogenase; ctrl, M-CSF treated controls; R+M, RANKL+M-CSF; R+M+T, RANKL+M-CSF+T-614 (iguratimod).

\section{Discussion}

Postmenopausal osteoporosis is an important clinical issue, which affects $50 \%$ of women over age 45 years (24). Exploring an agent to mitigate this problem effectively and safely will meet the needs of postmenopausal osteoporosis patients. In our previous study we observed that iguratimod reduced osteoclasts formation and bone destruction in the Walker 256 


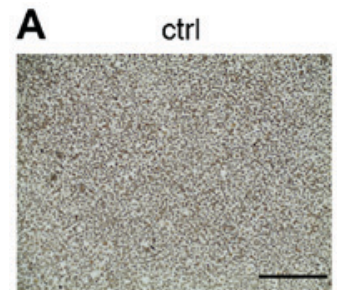

RANKL

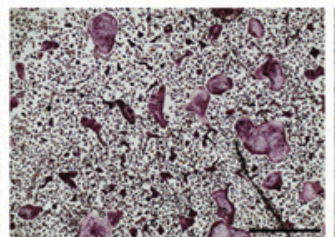

RANKL+T-614

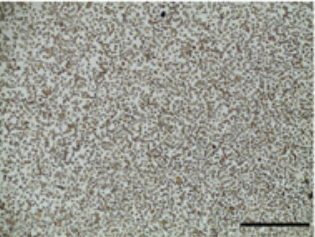

RANKL+BRL

$R A N K L+B R L+T-614$
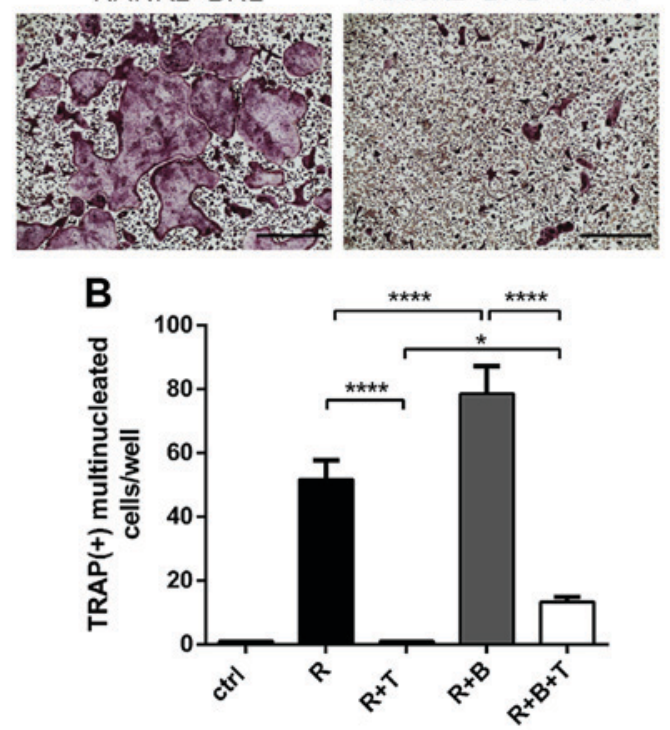

Figure 6. Rosiglitazone partly reverses the inhibitory effect of iguratimod on osteoclastogenesis. TRAP staining was performed (A) and TRAP ${ }^{+}$multinucleated cells were counted (B). The experiments were repeated 3 times independently. Data are presented as means \pm SD. Scale bar, $400 \mu \mathrm{m}$. ${ }^{*} \mathrm{P}<0.05,{ }^{* * * * *} \mathrm{P}<0.0001$. ctrl, M-CSF treated controls; R, RANKL; T, iguratimod (T-614); B, rosiglitazone (BRL).

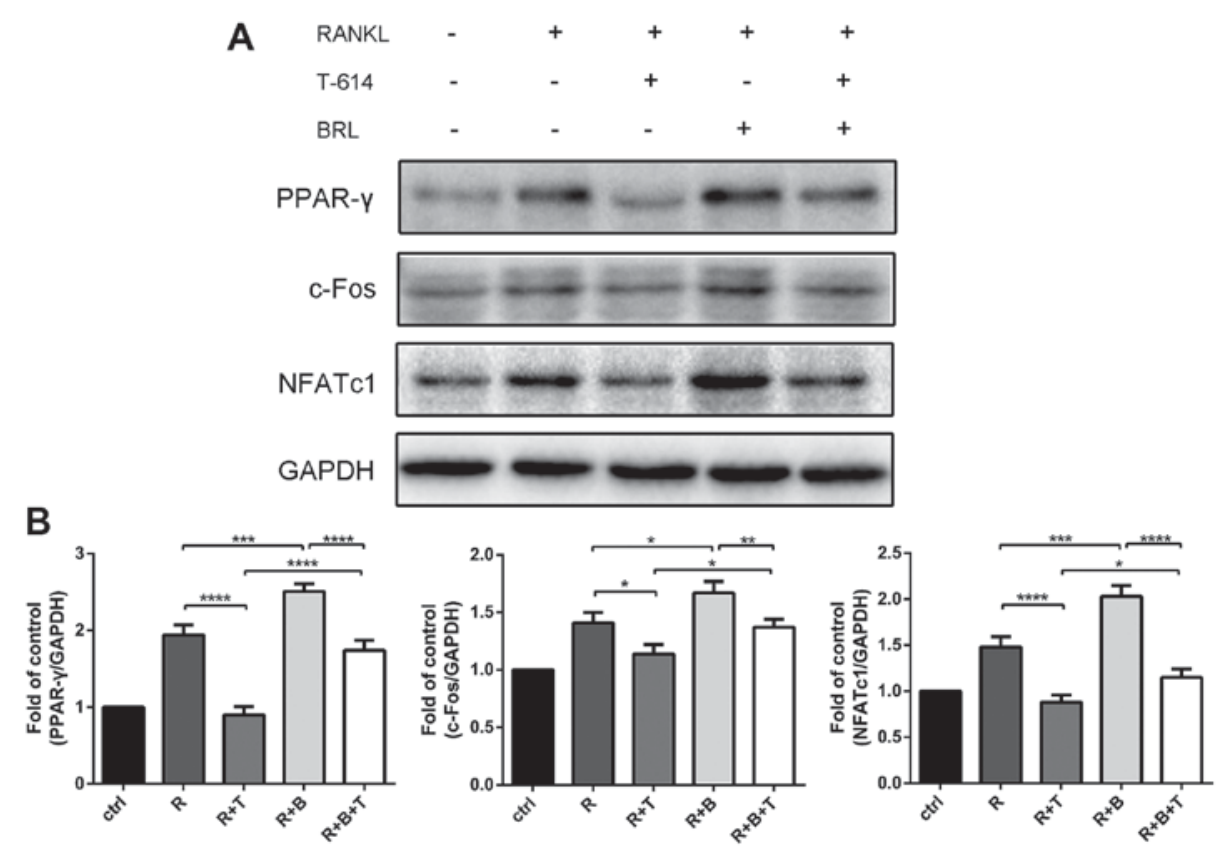

Figure 7. Iguratimod blocks PPAR- $\gamma / \mathrm{c}-$ Fos signaling. Proteins were extracted, and the protein expression levels of PPAR- $\gamma, \mathrm{c}-\mathrm{Fos}$ and NFATc1 were detected (A) and quantified (B). The experiments were repeated 3 times independently. Data are presented as means $\pm \mathrm{SD}$. ${ }^{*} \mathrm{P}<0.05,{ }^{* * *} \mathrm{P}<0.01,{ }^{* * * *} \mathrm{P}<0.001,{ }^{* * * * *} \mathrm{P}<0.0001$. PPAR- $\gamma$, peroxisome proliferator-activated receptor- $\gamma$; GAPDH, glyceraldehyde 3-phosphate dehydrogenase; ctrl, M-CSF treated controls; R, RANKL; $\mathrm{T}$, iguratimod (T-614); B, rosiglitazone (BRL).

rat mammary gland carcinoma cells induced bone cancer pain model (25). Other studies showed that mammary carcinoma cells are not bone-resorbing cells, but they stimulate osteocytes to express RANKL $(26,27)$. Over expression of RANKL may enhance osteoclasts formation and bone destruction. Considering the similar roles osteoclasts play in malignant 
and benign bone resorption, we hypothesized that iguratimod may also have therapeutic effects in benign bone metabolism diseases such as postmenopause osteoporosis.

In postmenopausal osteoporosis patients, dysfuction of ovaries induces estrogen deficiency and subsequently leads to the over expression of RANKL, which contributes to excessive osteoclastogenesis and trabecular bone loss (5). Our rationale to use ovariectomized mice as animal models in this study is based on reports that all major characteristics of bone loss associated with estrogen deficiency in humans can be mimicked in ovariectomized mice $(4,28)$. Our data showed that ovariectomy in mice led to trabecular bone loss, characterized by decreased BV/TV, Tb.N and increased SMI, Tb.Sp. Treatment of iguratimod significantly mitigated increased osteoclasts formation and increased serum levels of CTX-I, whereas these osteoporotic effects were significantly alleviated by treatment with iguratimod. These results suggested that iguratimod may be a therapeutic agent for OVX-induced bone loss. Taking into account the vital role of RANKL in postmenopausal bone loss, we presumed that targeting RANKL-induced osteoclastogenesis may be a reasonable explanation for the therapeutic effects of iguratimod in OVX mice.

Stimulation of RANKL facilitates the activation of c-Fos, which subsequently contributes to the induction and auto-amplification of NFATc1 (9). A previous report showed that stimulation of RANKL failed to increase NFATc1 levels in c-Fos deficient cells (29). Finally, NFATc1 activates osteoclast marker genes and promotes the formation and function of osteoclasts (30). Consistent with the in vivo study, our data showed that iguratimod could inhibit RANKL-induced osteoclasts formation and bone resorption activity of BMMCs in a dose-dependent manner. In addition, iguratimod drastically suppressed RANKL-mediated expression of NFATc1 and subsequently suppressed the activation of a number of osteoclast marker genes, including MMP-9 and cathepsin K, which can directly degrade collagens in demineralized hard tissues (11). Taken together, targeting RANKL-induced osteoclastogenesis may be a reasonable explanation for the therapeutic effects of iguratimod in OVX mice.

Considering the increased expression of PPAR $-\gamma$ in bone marrow of postmenopausal osteoporosis patients and ovariectomized animals $(31,32)$, the association between iguratmod and PPAR- $\gamma$ should be noticed. Although known as a key regulator of adipogenesis, the role of PPAR- $\gamma$ in osteoclastogenesis is also well characterized. A previous study showed that thiazolidinediones (PPAR- $\gamma$ agonists) may cause increased bone resorption in mice and rats (33). PPAR- $\gamma$-deficient mice suffer from impaired osteoclasts function and osteopetrosis caused by a direct reduction of c-Fos activation (16). In the present study, we revealed that iguratimod suppressed RANKL-induced osteoclast formation and treatment with rosiglitazone could partly reverse this inhibitory effect. Consistently, iguratimod also suppressed RANKL-induced expression of PPAR- $\gamma$, c-Fos and NFATc1. Treatment of rosiglitazone partly reversed the inhibitory effect of iguratimod. Thus, the prevention of RANKL-mediated bone resorption by iguratimod could also be attributed to the suppression of NFATc1 expression via blocking the PPAR- $\gamma / \mathrm{c}-$ Fos pathways.

Previously, Gan et al reported that iguratimod suppresses RANKL-induced osteoclasts differentiation and migration in RAW264.7 cells via NF- $\kappa \mathrm{B}$ and MAPK pathways (34). Wang et al also showed the effect of iguratimod on RANKL and OPG expression in serum and IL- $1 \beta$-induced fibroblast-like synoviocytes from patients with rheumatoid arthritis (35). These studies both implied the relationship between iguratimod and osteoclastogenesis in rheumatoid arthritis. Differently, our study verified that iguratimod could suppress osteoclasts formation and bone loss in postmenopausal osteoporosis animal models. In addition, RAW264.7 cells were derived from leukemia cells, our study further confirmed the effect of iguratimod on RANKL-induced osteoclastogenesis and the underlying mechanisms in primary BMMCs. Though NF- $\kappa \mathrm{B}$ and MAPK pathways play important roles in RANKL-induced osteoclasts differentiation, PPAR- $\gamma$ is also essential in RANKL-induced osteoclastogenesis through direct regulation of $\mathrm{c}-\mathrm{Fos}$ expression $(8,16)$. Considering the increased expression of PPAR $-\gamma$ in bone marrow of postmenopausal osteoporosis patients and ovariectomized animals $(31,32)$, inhibition of osteoclastogenesis via blocking the PPAR- $\gamma / \mathrm{c}-$ Fos pathway may contribute to the therapeutic effects of iguratimod in postmenopausal osteoporosis animal models.

In conclusion, the present study, to our knowledge, is the first to demonstrate that iguratimod can prevent ovariectomy-induced bone loss and that iguratimod can inhibit PPAR- $\gamma / \mathrm{c}-$ Fos pathway in RANKL-induced osteoclastogenesis. Considering the lifelong need to treat osteoporosis and that iguratimod is well-tolerated in long-term use (36), the anti-osteoclastogenic activity of iguratimod under clinical settings should be addressed in future.

\section{Acknowledgements}

This study was supported by grants from the National Nature Science Foundation of China (nos. 81572094 and 81371915).

\section{References}

1. Boyle WJ, Simonet WS and Lacey DL: Osteoclast differentiation and activation. Nature 423: 337-342, 2003.

2. Seeman E and Delmas PD: Bone quality-the material and structural basis of bone strength and fragility. N Engl J Med 354: 2250-2261, 2006.

3. An J, Yang H, Zhang Q, Liu C, Zhao J, Zhang L and Chen B: Natural products for treatment of osteoporosis: The effects and mechanisms on promoting osteoblast-mediated bone formation. Life Sci 147: 46-58, 2016.

4. Manolagas SC, O'Brien CA and Almeida M: The role of estrogen and androgen receptors in bone health and disease. Nat Rev Endocrinol 9: 699-712, 2013.

5. Onal M, Xiong J, Chen X, Thostenson JD, Almeida M, Manolagas SC and O'Brien CA: Receptor activator of nuclear factor $\kappa \mathrm{B}$ ligand (RANKL) protein expression by B lymphocytes contributes to ovariectomy-induced bone loss. J Biol Chem 287: 29851-29860, 2012.

6. Arai F, Miyamoto T, Ohneda O, Inada T, Sudo T, Brasel K, Miyata T, Anderson DM and Suda T: Commitment and differentiation of osteoclast precursor cells by the sequential expression of c-Fms and receptor activator of nuclear factor kappaB (RANK) receptors. J Exp Med 190: 1741-1754, 1999.

7. Lacey DL, Timms E, Tan HL, Kelley MJ, Dunstan CR, Burgess T, Elliott R, Colombero A, Elliott G, Scully S, et al: Osteoprotegerin ligand is a cytokine that regulates osteoclast differentiation and activation. Cell 93: 165-176, 1998.

8. Gu DR, Lee JN, Oh GS, Kim HJ, Kim MS and Lee SH: The inhibitory effect of beta-lapachone on RANKL-induced osteoclastogenesis. Biochem Biophys Res Commun 482: 1073-1079, 2017. 
9. Wagner EF and Eferl R: Fos/AP-1 proteins in bone and the immune system. Immunol Rev 208: 126-140, 2005.

10. Li C, Yang Z, Li Z, Ma Y, Zhang L, Zheng C, Qiu W, Wu X, Wang X, Li H, et al: Maslinic acid suppresses osteoclastogenesis and prevents ovariectomy-induced bone loss by regulating RANKL-mediated NF- $\kappa \mathrm{B}$ and MAPK signaling pathways. J Bone Miner Res 26: 644-656, 2011.

11. Takayanagi $\mathrm{H}$ : The role of NFAT in osteoclast formation. Ann $\mathrm{N}$ Y Acad Sci 1116: 227-237, 2007.

12. Conaway HH, Henning P, Lie A, Tuckermann J and Lerner UH: Activation of dimeric glucocorticoid receptors in osteoclast progenitors potentiates RANKL induced mature osteoclast bone resorbing activity. Bone 93: 43-54, 2016.

13. Kohno M, Aikawa Y, Tsubouchi Y, Hashiramoto A, Yamada R, Kawahito Y, Inoue K, Kusaka Y, Kondo M and Sano H: Inhibitory effect of T-614 on tumor necrosis factor-alpha induced cytokine production and nuclear factor-kappaB activation in cultured human synovial cells. J Rheumatol 28: 2591-2596, 2001.

14. Kawakami A, Tsuboi M, Urayama S, Matsuoka N, Yamasaki S, Hida A, Aoyagi T, Furuichi I, Nakashima T, Migita K, et al: Inhibitory effect of a new anti-rheumatic drug T-614 on costimulatory molecule expression, cytokine production, and antigen presentation by synovial cells. J Lab Clin Med 133: 566-574, 1999.

15. Du F, Lü LJ, Fu Q, Dai M, Teng JL, Fan W, Chen SL, Ye P, Shen N, Huang XF, et al: T-614, a novel immunomodulator, attenuates joint inflammation and articular damage in collagen-induced arthritis. Arthritis Res Ther 10: R136, 2008.

16. Wan Y, Chong LW and Evans RM: PPAR-gamma regulates osteoclastogenesis in mice. Nat Med 13: 1496-1503, 2007.

17. Luo Q, Sun Y, Liu W, Qian C, Jin B, Tao F, Gu Y, Wu X, Shen Y and Xu Q: A novel disease-modifying antirheumatic drug, iguratimod, ameliorates murine arthritis by blocking IL-17 signaling, distinct from methotrexate and leflunomide. J Immunol 191 4969-4978, 2013.

18. Fan H, Ji F, Lin Y, Zhang M, Qin W, Zhou Q and Wu Q: Electroacupuncture stimulation at CV4 prevents ovariectomy-induced osteoporosis in rats via Wnt- $\beta$-catenin signaling. Mol Med Rep 13: 2485-2491, 2016.

19. Chen Y, Wang XX, Zhao BJ, Bu J, Su YR and Zhang J: Effects of icariin on orthodontic tooth movement in rats. Int J Clin Exp Med 8: 8608-8616, 2015.

20. Koga T, Inui M, Inoue K, Kim S, Suematsu A, Kobayashi E, Iwata T, Ohnishi H, Matozaki T, Kodama T, et al: Costimulatory signals mediated by the ITAM motif cooperate with RANKL for bone homeostasis. Nature 428: 758-763, 2004.

21. Zhang Y, Guan H, Li J, Fang Z, Chen W and Li F: Amlexanox suppresses osteoclastogenesis and prevents ovariectomy-induced bone loss. Sci Rep 5: 13575, 2015.

22. Wang X, Zhu Y, Zheng S, Ni C, Zhao L, Liu C, Chen A and Xiao J: Amiloride inhibits osteoclastogenesis by suppressing nuclear factor- $\mathrm{\kappa} \mathrm{B}$ and mitogen-activated protein kinase activity in receptor activator of nuclear factor- $\kappa \mathrm{B}$-induced R AW264.7 cells. Mol Med Rep 11: 3451-3456, 2015.
23. Guan H, Zhao L, Cao H, Chen A and Xiao J: Epoxyeicosanoids suppress osteoclastogenesis and prevent ovariectomy-induced bone loss. FASEB J 29: 1092-1101, 2015.

24. Cline-Smith A, Gibbs J, Shashkova E, Buchwald ZS, Novack DV and Aurora R: Pulsed low-dose RANKL as a potential therapeutic for postmenopausal osteoporosis. JCI Insight 1: e88839, 2016.

25. Sun Y, Ye DW, Zhang P, Wu YX, Wang BY, Peng G and Yu SY: Anti-rheumatic drug iguratimod (T-614) alleviates cancer-induced bone destruction via down-regulating interleukin- 6 production in a nuclear factor- $\kappa \mathrm{B}$-dependent manner. J Huazhong Univ Sci Technolog Med Sci 36: 691-699, 2016.

26. Käkönen SM and Mundy GR: Mechanisms of osteolytic bone metastases in breast carcinoma. Cancer 97 (Suppl 3): S834-S839, 2003.

27. Roodman GD: Genes associate with abnormal bone cell activity in bone metastasis. Cancer Metastasis Rev 31: 569-578, 2012

28. Jilka RL, Hangoc G, Girasole G, Passeri G, Williams DC, Abrams JS, Boyce B, Broxmeyer H and Manolagas SC: Increased osteoclast development after estrogen loss: Mediation by interleukin-6. Science 257: 88-91, 1992.

29. Asagiri $\mathrm{M}$ and Takayanagi $\mathrm{H}$ : The molecular understanding of osteoclast differentiation. Bone 40: 251-264, 2007.

30. Ortega N, Behonick D, Stickens D and Werb Z: How proteases regulate bone morphogenesis. Ann N Y Acad Sci 995: 109-116, 2003.

31. Bu S, Chen Y, Wang S, Zhang F and Ji G: Treadmill training regulates $\beta$-catenin signaling through phosphorylation of GSK-3 $\beta$ in lumbar vertebrae of ovariectomized rats. Eur J Appl Physiol 112: 3295-3304, 2012.

32. Li GW, Xu Z, Chang SX, Nian H, Wang XY and Qin LD: Icariin prevents ovariectomy-induced bone loss and lowers marrow adipogenesis. Menopause 21: 1007-1016, 2014.

33. Sottile V, Seuwen K and Kneissel M: Enhanced marrow adipogenesis and bone resorption in estrogen-deprived rats treated with the PPARgamma agonist BRL49653 (rosiglitazone). Calcif Tissue Int 75: 329-337, 2004.

34. Gan K, Yang L, Xu L, Feng X, Zhang Q, Wang F, Tan W and Zhang M: Iguratimod (T-614) suppresses RANKL-induced osteoclast differentiation and migration in RAW264.7 cells via NF- $\kappa \mathrm{B}$ and MAPK pathways. Int Immunopharmacol 35: 294-300, 2016.

35. Wang XT, Li P, Xu TS, Ding R, Zhang X and Bi LQ: Effect of iguratimod and methotrexate on RANKL and OPG expression in serum and IL-1 $\beta$-induced fibroblast-like synoviocytes from patients with rheumatoid arthritis. Cell Mol Biol (Noisy-le-grand) 62: 44-50, 2016.

36. Okamura K, Yonemoto Y, Suto T, Okura C and Takagishi K: Efficacy at 52 weeks of daily clinical use of iguratimod in patients with rheumatoid arthritis. Mod Rheumatol 25: 534-539, 2015. 